Diagnostic Images

\title{
Iatrogenic disease. Lipoid pneumonia and liver angiosarcoma
}

\author{
Presented by L. Kreel \\ Newham General Hospital, London E13, UK.
}

A man aged 86 years presented with severe debility, loss of weight and appetite. He was known to have lipoid 'pneumonia' and had had thorotrast arteriography 20 years previously.

Computed tomographic (CT) scan was required to exclude malignancy.

\section{Comment}

In adults lipoid pneumonia is usually caused by the use of oily nose drops or mineral oil as a laxative. In both cases the oil droplets tend to congregate in the right main bronchus being in a more direct line with the trachea than the left main bronchus. The dorso-lateral segments of the upper lobe are involved if medication is taken before retiring for the night and basal segments in the morning medicators. Lipoid pulmonary masses can be mistaken for carcinoma macroscopically or granulomatous disease such as tuberculosis or sarcoidosis on histology as it provokes a marked fibroblastic reaction with multinucleate giant cells to form granulomas. On CT low attenuation indicates the lipid nature of the 'solid' mass unlike a large bronchial carcinoma. Thorotrast was used in the period 1925-1950 as an intravascular contrast agent particularly for cerebral angiography because of its high density and low immediate toxicity. However, malignant transformation was subsequently recognized as a later complication occurring on average after a latent period of 20 years. Angiosarcoma of lymph nodes, spleen and liver occurred as well as cholangiocarcinoma, lymphoma and bronchial carcinoma.

Angiosarcomas of the liver are known to be caused by arsenical preparations particularly pesticides and polyvinyl chloride used in the manufacture of plastics as well as by thorotrast.

The radiological features of thorotrast are pathognomonic as it accumulates in the reticuloendothelial system producing an extremely dense spleen, liver and lymph nodes. Malignancy can be diagnosed when the organ becomes less dense, enlarges and contains filling defects. Ascites is a secondary feature of the malignancy.

\section{References}

BOYD, J.T., LANGLANDS, A.O. \& MACCABE, J.J. (1968). Long-term hazards of thorotrast. British Medical Journal, 2, 517 .

KENNEDY, J.D., COSTELLO, P., BALIKIAN, J.P. \& HERMAN, P.G. (1981). Exogenous lipoid pneumonia. American Journal of Roentgenology, 136, 1145.

POPPER, H., THOMAS, L.B., TELLES, N.C., FALK, H. \& SELIKOFF, I.J. (others) (1978). Development of hepatic angiosarcoma in man induced by vinyl chloride, thorotrast and arsenic. Comparison with cases of unknown aetiology. American Journal of Pathology, 92, 349. 


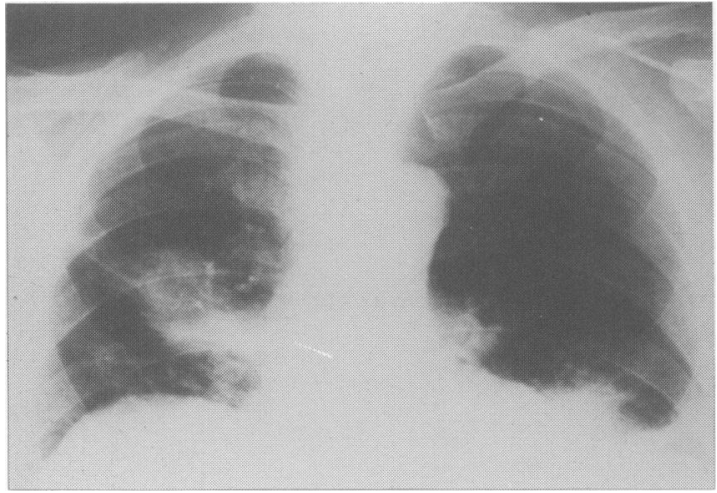

Figure 1 The chest radiograph shows a large mass adjacent to the right hilum, another smaller mass at the left hilum and $a$ third at the left base. The lateral border of the right sided mass has a 'whiskery' appearance of marginal fibrosis and the mass for its size has a low density in keeping with a lipoid granuloma. The left sided mass lesions, though smaller, have similar appearances.

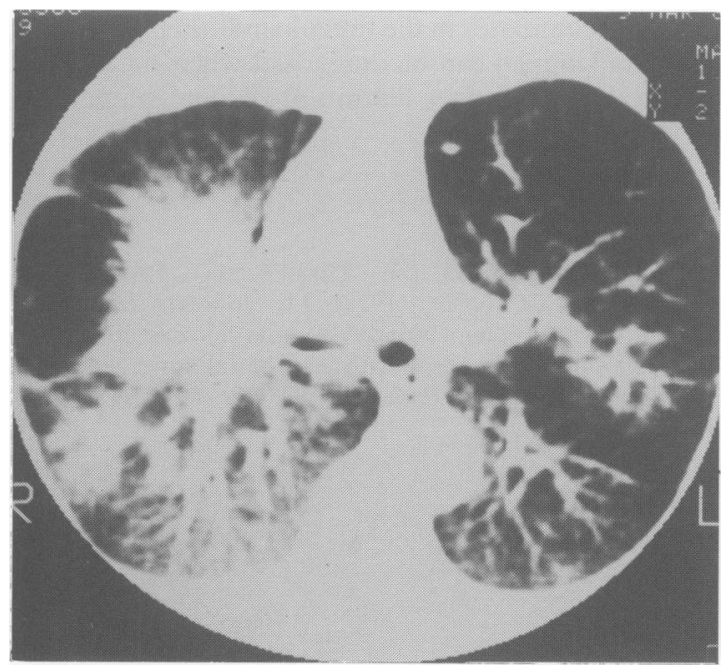

Figure 2 CT scan at lung settings shows the large hilar mass with irregular 'whiskery' lateral margin and fibrous strands reaching the pleura. A smaller mass is present postero-laterally, another at the left hilum and an irregular nodule lying posteriorly on the left.

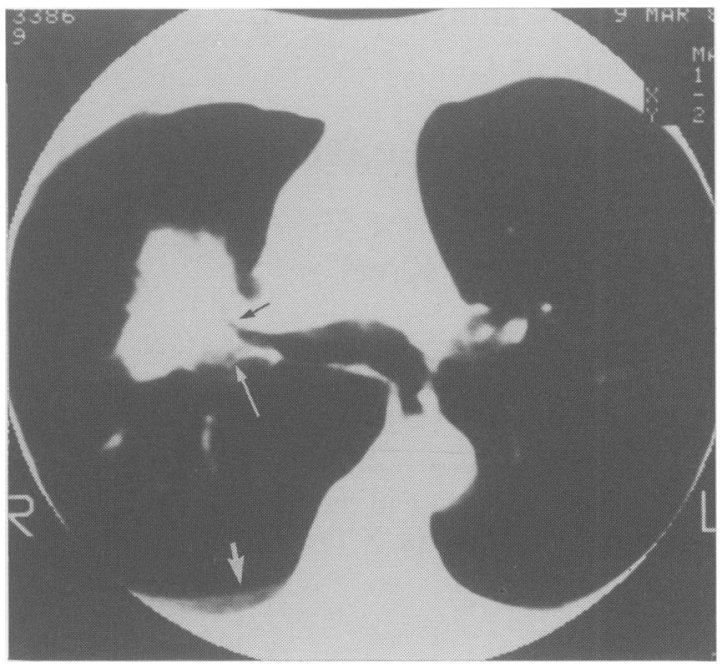

Figure 3 Scan to show right main bronchus leading to the right upper lobe bronchus and giving off the anterior segmental and posterior segmental bronchi (arrows) which appear markedly narrowed. The attenuation value taken within the centre of the mass to avoid the partial volume effect, was well below that of soft tissues indicating its lipoid content ( -60 Hounsfield units). There is also a very small right pleural effusion (arrow).

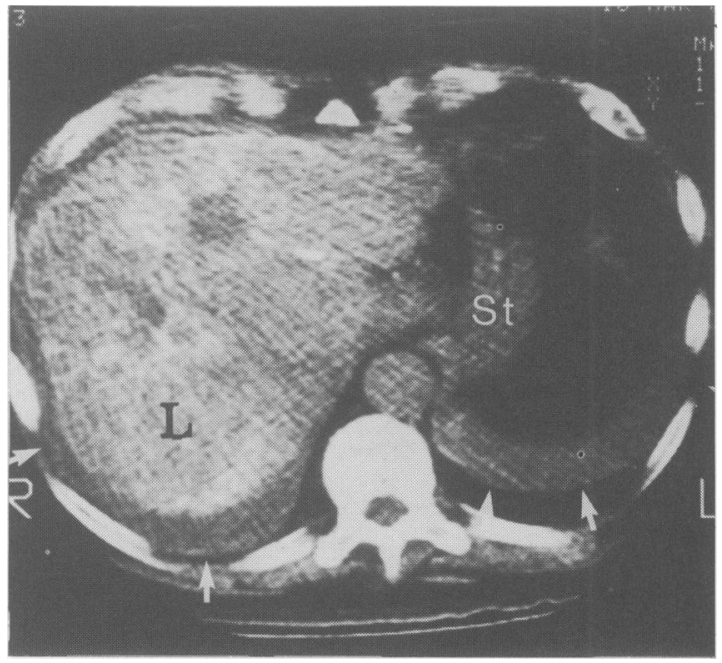

Figure 4 Section through the upper part of the liver (L) and body of the stomach (St). The liver is abnormally dense with multiple small rounded areas of lower attenuation. Ascites is present (arrows) around the liver and anterior to the left hemidiaphragm. 


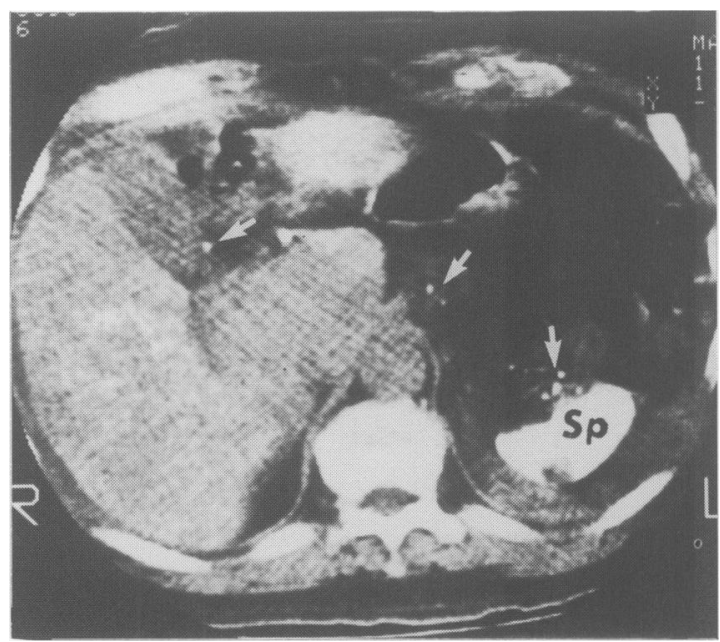

Figure 5 The spleen (Sp) appears white due to the very high density of retained thorotrast and shows a small nodule posteriorly with ascites lying between the spleen and the left hemidiaphragm. The small white high density nodules (arrows) are lymph nodes containing thorotrast. The high density liver has a very patchy appearance.

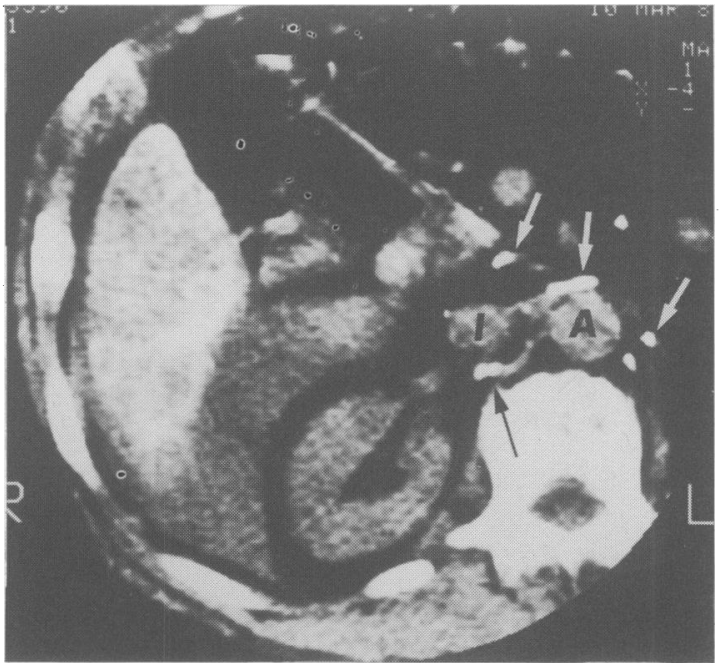

Figure 6 Caudal aspect of the right lobe of the liver is dense with round filling defects and surrounded by ascites posteriorly. Multiple contrast filled (thorotrast) lymph nodes around the aorta (A) and inferior vena cava (I). 